\title{
Analysis of technical and economic parameters of fusion power plants in future power systems
}

\author{
Inga M. Müller ${ }^{\mathrm{a}, *}$, Matthias Reich ${ }^{\mathrm{b}}$, Felix Warmer ${ }^{\mathrm{c}}$, Hartmut Zohm ${ }^{\mathrm{b}}$, Thomas Hamacher ${ }^{\mathrm{a}}$, Sibylle Günter ${ }^{\mathrm{b}}$, \\ ${ }^{a}$ Chair of Renewable and Sustainable Energy Systems, Technical University of Munich, Arcisstr. 21, 80333 Munich, Germany \\ ${ }^{b}$ Max-Planck-Institute for Plasma Physics, Bolzmannstr. 2, 85748 Garching, Germany \\ ${ }^{c}$ Max-Planck-Institute for Plasma Physics, Wendelsteinstr. 1, 17491 Greifswald, Germany
}

\begin{abstract}
The European power system faces two main challenges: first, the increasing share of fluctuating energy sources in the power generation sector challenges the balance of generation and demand. Second, the expected shift towards using more electricity in the heating and transportation sectors leads to rising demand. To ensure both sufficient energy supply and long-term power system stability of the power system, fusion power is expected to play an important role in the future. This paper provides a sensitivity analysis of fusion power plant parameters in a power system with a large fraction of renewable energies. The results depend strongly on the underlying scenario assumptions, especially on future technical and economic improvements. Finally, the amount of fusion power investments depends on both demand level and relative costs of competing technologies.
\end{abstract}

Keywords: Fusion, Renewable Energies, Energy System Modeling

\section{Introduction}

A future European power system faces several challenges which are, inter alia, adequate supply of the increased electricity demand and integration of fluctuating energy sources. To reduce overall $\mathrm{CO}_{2}$ emissions, it becomes attractive to connect the heating and transportation sector to the power sector and the consequent shift towards using more electricity in those sectors also leads to an increased future electricity demand. Both the subsequent replacement of fossil power plants by emission free technologies like solar and wind power and the expansion of power plant capacity to fulfill additional demand increase the share of fluctuating energy sources in the power sector. Thus, the task of balancing generation and demand becomes more difficult. To ensure both sufficient energy supply and long-term stability of the power system, fusion power is expected to play an important role in the future due to its attractive properties, inherent safety and $\mathrm{CO}_{2}$ emission free operation.

This paper provides a sensitivity analysis of economic fusion power plant commitment in a power system with a large fraction of renewable energies as strived for towards the end of the 21 st century.

\section{Method}

For our sensitivity analysis, a linear optimization model urbs [1] is applied considering expansion and dispatch planning. urbs represents the European power system and includes power

\footnotetext{
${ }^{*}$ Corresponding author

Email address: inga.mueller@tum.de (Inga M. Müller)
}

plants, energy storages and transmission lines. The model optimizes the system costs while covering one year of operation with an hourly time resolution. The model comprises $34 \mathrm{Eu}-$ ropean countries which are aggregated to 10 groups. Based on three main scenarios more than 250 sub-scenarios were defined to determine the structure of a future power system in a greenfield approach with urbs.

\subsection{Model description}

urbs is a linear multi-commodity unit planning and commitment model which minimizes the annual total system costs. It is suitable and regularly applied for expansion planning. The structure of the model is given by one objective function and various constraints. The structure of the model is given by:

$$
\begin{aligned}
\min _{x} c^{T} x & \\
\text { s.t. } & A x \leq b
\end{aligned}
$$

The objective function comprises investment, fixed, variable and fuel costs of all processes, storages and transmission lines. The total costs are to be minimized. The constraints represent physical restrictions of the model such as energy balance, upper bounds for component expansions and emission limits.

\subsection{Scenarios and assumptions}

For the analysis three main scenarios min, mid, max are defined. All scenarios represent a future system with varied electrical efficiency improvements and technology developments leading to three variable electricity demands ( $m i n$, mid, max). The costs of the system components are expected to be low for 
the min, high for the max and in between for the mid scenario. Through the link between demand and costs developments, a large scenario framework is covered by reducing the number of scenarios Thus, from the perspective of fusion power, the $\mathrm{min}$ scenario represents a worst-case scenario as demand and costs of competing technologies are low whereas the max scenario represents a best-case scenario. Additionally, the fundamental market link between demand and prices as well as the decreasing of suitable locations for wind and solar power plants are considered: with increasing demand, also unsuitable locations for renewable energies with lower full load hours are chosen resulting in relatively high costs.

Complementing the three main scenarios, four sub-scenarios are created featuring renewable and additional technologies as follows: (1) An emission-free scenario with fission and fusion power plants: OCO2_wNuc. (2) An emission-free scenario with fusion power plants only: OCO2_woFis. (3) A scenario where fossil, fission and fusion technologies are used at a $5 \%$ $\mathrm{CO}_{2}$ emission level (compared to 1990): 5CO2_wNuc. (4) A renewables-only scenario $(R E S)$ serves as a reference, where each of the three viable scenarios can be compared against (see Table 1).

Further, for expansion planning with urbs two parameters are varied: (1) Investment costs of fusion power plants range from 3.000 to $10.000 € / \mathrm{kW}_{e l}$. Fixed costs are assumed proportional to the investment costs at a quota of $2 \%$ per annum (see [2]). (2) Variable costs of fusion power plants range from 2,5 to $7,5 € / \mathrm{MWh}_{e l}$. Making assessments about technical and economic parameters of fusion power plants is highly uncertain. Based on known parameters of fission power plants [2], the parameter ranges for future fusion technology were set by an expert group [8]. Moreover, the parameter ranges relate to an established and well-developed fusion power technology. The large range of fusion investment costs is defined precisely to address the identification of investment thresholds caused by different system conditions.

In all scenarios the expansion of renewable energies (RES), fusion and fission power plants is limited. The expansion limit of RES depends on regional factors. The aggregated capacity limit for all 34 European countries is 2 TW for wind onshore, $125 \mathrm{GW}$ for wind offshore, $24 \mathrm{TW}$ for solar, $272 \mathrm{GW}$ for hydro and 4930 TWh for biomass.

With regard to fusion, it is conservatively assumed that until 2100 not more than $50 \mathrm{GW}_{e l}$ can be installed. The conservative limit includes delayed market maturity, long construction periods and limited material availability for fusion power plants. Assuming technological maturity of fusion power plants in 2060, the potential construction period for fusion power plants is 40 years. Due to similarities in thermal and electrical power output between fusion and fission power plants of generation III, the current market penetration rate of fission power plant generation III is taken as a guideline. According to [2], there could be $40 \mathrm{GW}_{e l}$ in 2030 (approx. 40 years construction period), $80 \mathrm{GW}_{e l}$ in 2040 (approx. 50 years) and $1200 \mathrm{GW} e l$ in 2050 (approx. 60 years). For our conservative approach, including possible delays due to the novel plant core, longer construction periods and resource limitations, we determine a

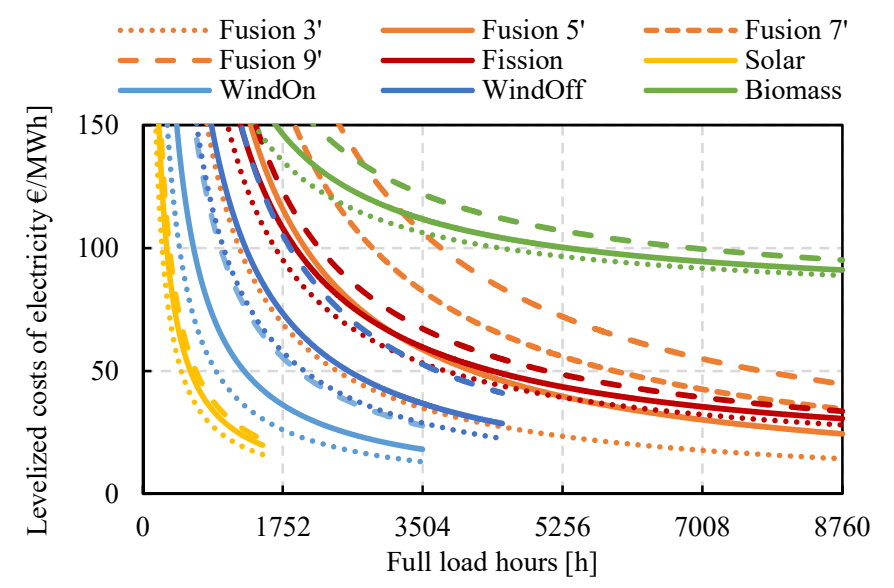

Figure 1: Levelized costs of electricity including investment, fixed, variable and fuel costs (low, medium, high prices), based on [2] and four fusion investment cost values between 3.000 and $9.000 € / \mathrm{kW}_{e l}$.

Table 1: Overview of the scenario structure containing three main and four sub-scenarios applied in the analysis.

\begin{tabular}{|c|c|c|c|c|c|}
\hline demand & $\mathrm{CO}_{2}$ & Fusion & Fission & Fossil & \\
\hline \multirow{4}{*}{$\begin{array}{l}\min \\
\max \end{array}$} & $0 \%$ & $X$ & $\mathrm{X}$ & - & (wNuc) \\
\hline & $0 \%$ & X & - & - & (woFis) \\
\hline & $5 \%$ & $\mathrm{X}$ & $\mathrm{X}$ & $\mathrm{X}$ & (wAll) \\
\hline & $0 \%$ & - & - & - & (RES) \\
\hline
\end{tabular}

capacity limit of $50 \mathrm{GW}_{e l}$ in 2100 .

The limit for fission power plants is set to the capacity installed in 2015 except for countries which back out of the fission energy program where it is set to zero. In total the maximum installable capacity of fission power plants in Europe is $117 \mathrm{GW}$. The costs of system components, including power plants, pump and battery storages, and transmission lines, are based on [2] and [3] which state different costs developments until 2050. Due to missing sources predicting costs until 2100, it is assumed that between 2050 and 2100 no significant costs changes occur and that uncertainty is covered by different costs scenarios (see three main scenarios). The grid topology and the expansion limits for geothermal power, hydro power and wind offshore are based on [4]. The limits for wind onshore and solar power as well as the capacity factors, which cover one year with an hourly resolution and thus represent daily and seasonally fluctuations, are calculated with an internal tool based on [5] and [6]. The calculation in both tools depends on regional measured climate data. The limit for energy from biomass is extracted from [7]. To calculate the specific demand a separate tool has been developed [9].

The optimization model represents a central planner which makes economically rational decisions. Thus, social and political reservation against, e.g., grid expansion or storages are not considered. Table 1 summarizes the key elements of the scenarios whereas Figure 1 displays total annual costs of selected technologies for three main scenarios. 


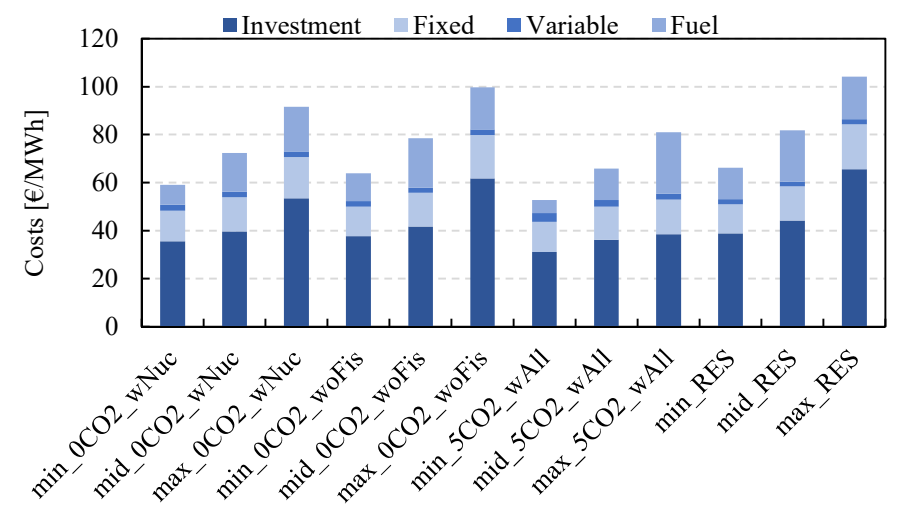

Figure 2: System costs of selected scenarios. The investment costs of fusion power plants are $5.000 € / \mathrm{kW}_{e l}(0,8-1,05$ compared to fission).

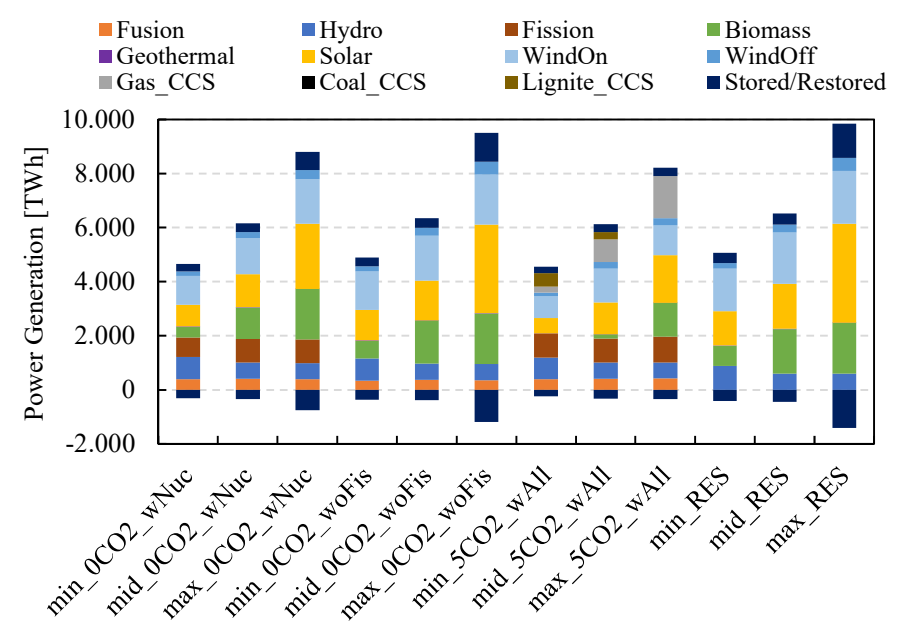

Figure 3: Power generation and storage of the selected scenarios.

\section{Results}

The results of the analysis are presented in two sections. In the first section we present an overview of the overall system results based on urbs. In the second section, we present results based on the variation of economic investment parameters. All results are sensitive to the underlying scenario assumptions.

\subsection{Overview of overall system}

The first part of our analysis involves all combinations of demand profiles ( $\min$, mid, max) with all four power system scenarios. In total, 12 scenarios describing 12 separate optimization problems are compared to investigate the impact of different parameters on cost optimal system configurations. Figure 2 displays the comparison of the total system costs including investment, fixed, variable and fuel costs summarized for all power plants, storages and transmission lines. The costs are normalized to the corresponding demand (specific costs). It is evident that the demand represented in the three main scenarios strongly affects the cost variation on both system costs and specific costs level. However, the impact of increasing power plants and storage costs - also included in the main scenarios on the resulting system cost difference is relatively small (share lower than $2 \%$, see also Figure 1). The system costs are highest when only RES are allowed. With the addition of fusion power plants, system costs are lowered by $3,5 \%$ ( $\min$ ) to $4,2 \%$ (max) according to the optimization model. If also fission and fossil power plants are allowed $\left(5 \% \mathrm{CO}_{2}\right)$ the costs can be reduced by up to $22 \%$. The cost differences are mainly caused by the changes in investment and fuel costs which correlate with the installed capacity and the supplied power.

When comparing different scenarios, it is observed that an increase in demand is always fulfilled by an expansion of wind and solar power plants as fusion and fission power plants have already reached their capacity limits. All emission-free power systems highly depend on solar (44-64\%) and wind power (22$32 \%$ ). With the availability of fusion power plants, the optimal installed capacity of solar is lower by up to $348 \mathrm{GW}(11 \%$ decrease) and the capacity of wind is lower by up to $154 \mathrm{GW}$ (16\% decrease) compared to the RES scenario. In 5CO2_wAll, where also fission and fossil power plants are considered, the optimal installed capacities of renewables are lower by up to 1,6 TW (50\% decrease) and $558 \mathrm{GW}$ (53\% decrease), respectively. Besides expanding wind and solar power plants, investments in storage power as well as in storage capacity increases with growing demand. Moreover, the fewer conventional power plants exist, the higher is the resulted storage requirement (188 GW/825 GWh up to $772 \mathrm{GW} / 4540 \mathrm{GWh})$. If fusion power plants are added to a power system based only on RES, the storage capacity (and storage power) is reduced by $8 \%(6 \%)$ in the min scenario and up to $15 \%(14 \%)$ in the $\max$ scenario.

Due to curtailment and generally low full load hours of renewables compared to base load plants, the actual contribution of power generated by the systems as shown in Figure 3 is more evenly distributed. In a RES based power system solar and wind generation represents a share of $27-43 \%$ or rather $28-38 \%$. When fusion power plants are added the share of both technologies decreases to $19-30 \%$ and to 24-28\%. Independent of the sub-scenario fusion always represents a share of 4-9\%, mainly as a result of conservatively chosen fusion capacity limits.

\subsection{Sensitivity Analysis: Investment Parameters}

Based on the three main scenarios and first three subscenarios $(0 \%$ emission with and without fission power plants, $5 \%$ emission) a comprehensive sensitivity analysis regarding investment and variable costs has been conducted. Thus, critical fusion investment limits are identified, at which the optimal system configuration changes significantly.

Figure 4 shows the installed capacity of fusion power as function of investment costs (x-axis) and variable costs (line type) for each scenario-sub-scenario combination. In scenarios with low demand and system costs, the system reacts more sensitive to an increase in fusion investment and variable costs. This sensitivity is reduced in scenarios, where fission power plants are excluded as an alternative power source. In the emission free scenario fusion is installed to the set expansion limit up to costs of $5.000 € / \mathrm{kW}_{e l}$ (min), $7.000 € / \mathrm{kW}_{e l}$ (mid) and (more than) $10.000 € / \mathrm{kW}_{e l}$ (max). At a capacity utilization of $30 \mathrm{GW}_{e l}(60 \%$, representing the share of installed capacity compared to maximum installable capacity) investment costs 


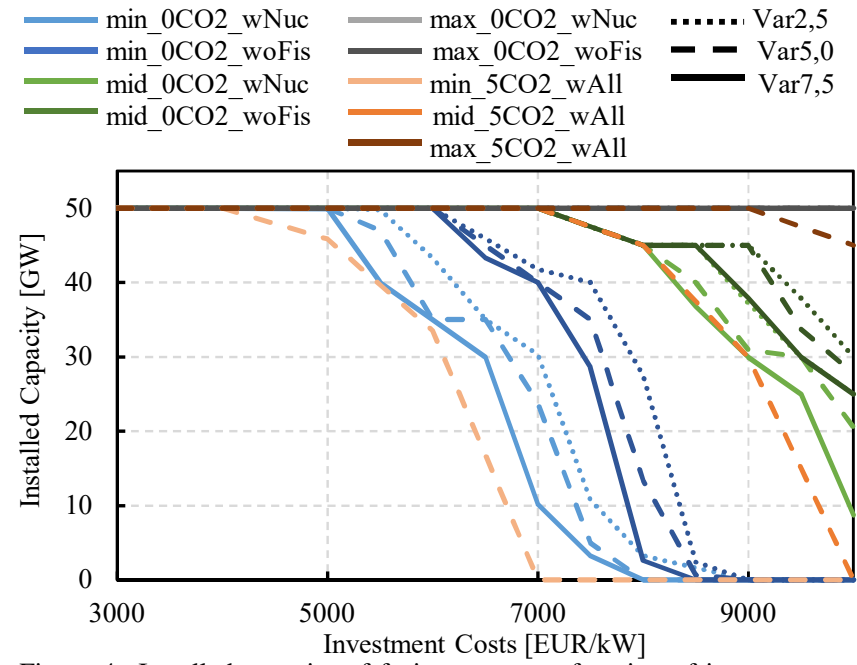

Figure 4: Installed capacity of fusion power as function of investment costs (x-axis) and variable costs (line type) for each scenario combination. Three main and three variable-costs scenarios are considered for 0CO2_wNuc and OCO2_woFis systems whereas $w \_A l l$ is only presented for three main scenarios and one variable costs-case.

up to $6.500 € / \mathrm{kW}_{e l}\left(9.000 € / \mathrm{kW}_{e l}\right)$ are part of the optimal solution. In the scenario $5 \%$ emissions, costs limits are shifted mainly in the min scenario by a maximum of $1.000 € / \mathrm{kW}_{e l}$. In other scenarios the deviation is only significant at investment costs above $9.000 € / \mathrm{kW}_{e l}$.

A country level analysis shows that countries with good wind sites and therefore lower specific costs for wind power prefer wind power over fusion power already at lower (fusion power) market prices whereas states with better solar sites would build fusion power also at slightly higher absolute costs. Costs thresholds, representing breakeven cost of fusion, depend on the specific scenario. In figure 4 these costs are indicated by curve progressions. In a min scenario, including fission and fusion power, breakeven costs of countries with good wind sites are $5.500 € / \mathrm{kW}_{e l}$ whereas in the corresponding max scenario the costs are higher than $10.000 € / \mathrm{kW}_{e l}$.

\section{Conclusion}

The results of this sensitivity analysis provide first indications for investment decisions of fusion power plants in a future European power system of 2100 . The analysis bases on a large scenario framework with three main scenarios (worst-case ( min), mid-case ( mid) and best-case (max)) with varied system costs and demand levels.

The first analysis (3.1) shows that the effect of fusion on system cost savings are noticeable but low compared to fossil based systems with $5 \%$ emissions. Compared to the RES scenario, system costs are reduced by $4 \%$ when fusion power plants are included, whereas in systems with additional fission and fossil power plants the costs are lowered by $22 \%$.

The second part 3.2) indicates that fusion investment decisions depend on system conditions which are the costs of competing system components and in particular the level of demand. Up to $5.000 € / \mathrm{kW}_{e l}$ fusion is fully expanded in all scenarios except the min scenario with emissions. Even at investment costs of
$8.000 € / \mathrm{kW}_{e l}$, the fusion expansion rate is larger than $90 \%$ for all mid and max scenarios. Moreover, for fusion expansion, the emission limit and thus the fossil power plant option is only significant at rather high investment costs. The demand levels have stronger impact on the feasibility of fusion power at a certain cost than climate goals (e.g. min_0CO2_wNuc vs. min 5_CO2_wAll vs. mid_0CO2_wNuc at $6000 € / \mathrm{kW}_{e l}$ ).

As stated earlier, the findings are valid only under the set assumptions. As forecasts about social, economic and technological developments are not possible, a broad scenario framework is defined. Despite the broad covered range, uncertainties remain, in particular for the development of fusion power plants. Hence, further analysis, e.g., scenarios without capacity limits should check the robustness of the results. To better quantify effects of increasing system costs and different demand levels, additional investigations should be conducted focusing on the separation of costs and demand effects. Finally, a more detailed power system and better representation of fusion power plants can provide further input, e.g., in the case of identification of fusion parameters which switch the operation mode from a pure base load to an economically operating flexible power plant.

\section{References}

[1] J. Dorfner, M. Dorfner, K. Schönleber, S. Candas, S. Müller et al.: urbs. https://zenodo.org/record/1228851 2018

[2] European Commission: ETRI 2014: Energy Technology Reference Indicator projections for 2010-2050. 2014

[3] M. Fürsch, S. Hagspiel, C. Jägermann, S. Nagl, D. Lindenberger et al.: Roadmap 2050 a closer look: Cost-efficient RES-E penetration and the role of grid extensions. 2011

[4] European Commission: Energy Roadmap 2050 - SEC(2011) 1565 final Part 1/2. 2011

[5] K. Siala, J. Stich: Estimation of the PV potential in ASEAN with a high spatial and temporal resolution. Renewable Energy. Vol. 88. pp. 445-456. 2016

[6] K.A. Janker: Aufbau und Bewertung einer für die Energiemodellierung verwendbaren Datenbasis an Zeitreihen erneuerbarer Erzeugung und sonstiger Daten. Doctoral Thesis. Technical University of Munich. 2015

[7] B. Elbersen, I. Startisky, G. Hengeveld, M.-J. Schelhaas, H. Naeff et al.: Atlas of EU biomass potentials. 2012

[8] Max-Planck-Institute for Plasma Physics, Karlsruhe Institute of Technology and Chair of Renewable and Sustainable Energy Systems, personal communication, May 2018

[9] D. Roßmann: Entwicklung eines Python Tools zur automatisierten Berechnung des elektrischen Endenergiebedarfs unter Berücksichtigung des Wärme- und Verkehrssektors. Master Thesis. Technical University of Munich. 2018 